\title{
A National French consensus on gene lists for the diagnosis of myopathies using next-generation sequencing
}

\author{
Martin Krahn ${ }^{1,2} \cdot$ Valérie Biancalana $^{3} \cdot$ Mathieu Cerino $^{1,2} \cdot$ Aurélien Perrin $^{4,5}$ - Laurence Michel-Calemard ${ }^{6}$. \\ Juliette Nectoux ${ }^{7}$. France Leturcq ${ }^{7}$ - Céline Bouchet-Séraphin ${ }^{8}$. Cécile Acquaviva-Bourdain ${ }^{9}$. \\ Emmanuelle Campana-Salort ${ }^{1,10}$ - Annamaria Molon ${ }^{10}$ - Jon Andoni Urtizberea ${ }^{11}$ • Frédérique Audic $^{1,10}$. \\ Brigitte Chabrol ${ }^{10}$ - Jean Pouget ${ }^{1,10}$. Roseline Froissart ${ }^{9} \cdot$ Judith Melki $^{12}$ - John Rendu ${ }^{13,14,15}$. François Petit $^{16}$. \\ Corinne Métay ${ }^{17} \cdot$ Nathalie Seta $^{8} \cdot$ Damien Sternberg $^{17} \cdot$ Julien Fauré ${ }^{13,14,15} \cdot$ Mireille Cossée $^{4,5}$
}

Received: 12 March 2018 / Revised: 17 October 2018 / Accepted: 25 October 2018 / Published online: 14 December 2018

(c) European Society of Human Genetics 2018

\begin{abstract}
Next-generation sequencing (NGS) gene-panel-based analyses constitute diagnosis strategies which are adapted to the genetic heterogeneity within the field of myopathies, including more than 200 implicated genes to date. Nonetheless, important interlaboratory diversity of gene panels exists at national and international levels, complicating the exchange of data and the visibility of the diagnostic offers available for referring neurologists. To address this issue, we here describe the initiative of the genetic diagnosis section of the French National Network for Rare Neuromuscular Diseases (Filière Nationale des Maladies Rares Neuromusculaires, FILNEMUS), which led to set up a consensual nationwide diagnostic strategy among the nine French genetic diagnosis laboratories using NGS for myopathies. The strategy is based on the determination of 13 clinical and/or histological entry-diagnosis groups, and consists for each group either in a successive NGS analysis of a "core gene list" followed in case of a negative result by the analysis of an "exhaustive gene list", or in the NGS analysis of a "unique exhaustive gene list".
\end{abstract}

These authors contributed equally: Valérie Biancalana, Mathieu Cerino, and Aurélien Perrin

Supplementary material The online version of this article (https:// doi.org/10.1038/s41431-018-0305-1) contains supplementary material, which is available to authorized users.

Martin Krahn

martin.krahn@univ-amu.fr

1 Aix Marseille Univ, Inserm, MMG, Marseille Medical Genetics Translational Neuromyology, Marseille, France

2 APHM, Hôpital Timone Enfants, Département de Génétique Médicale, Marseille, France

3 Laboratoire Diagnostic Génétique, Faculté de Médecine, CHRU, Nouvel Hôpital Civil, Strasbourg, France

4 CHRU Montpellier, Laboratoire de Génétique moléculaire, Montpellier, France

5 Université Montpellier, Laboratoire de Génétique de maladies rares, Montpellier, France

6 HCL, Centre de Biologie et Pathologie Est, UM Pathologies Endocriniennes, Rénales, Musculaires et Mucoviscidose, Bron, France

7 APHP, Laboratoire de génétique et biologie moléculaires, HUPC Cochin, Paris, France

8 APHP, Département de Génétique, Hôpital Bichat Claude

\section{Introduction}

The genetic heterogeneity of myopathies constitutes a challenge for diagnostic strategies, with more than 200

Bernard, Paris, France

9 Service Maladies Héréditaires du Métabolisme et Dépistage Néonatal, Centre de Biologie et de Pathologie Est - CHU de Lyon, Lyon, France

10 APHM, Hôpital Timone, Centre de référence des maladies neuromusculaires et de la SLA, Marseille, France

11 APHP, Hôpital Marin, Centre de compétence neuromusculaire Hendaye, Hendaye, France

12 Inserm UMR-1169, Université Paris Sud, Paris, France

13 Centre Hospitalier Régional Universitaire de Grenoble, Hôpital Michallon, Biochimie Génétique et Moléculaire, Grenoble, France

14 Univ Grenoble Alpes, Grenoble Institut des Neurosciences, GIN, Grenoble, France

15 Inserm U1216, Grenoble, France

16 APHP, Laboratoire de Génétique moléculaire, GH Antoine Béclère, Clamart, France

17 APHP, Centre de génétique moléculaire et chromosomique, Hôpital Pitié-Salpêtrière, Paris, France 
implicated genes reported to date (Gene Table of Neuromuscular Disorders, http://www.musclegenetable.fr) [1]. As for other monogenic diseases, the implementation of nextgeneration sequencing (NGS) for diagnostic purposes allowed for an unprecedented increase in mutational screening capacities in the field of myopathies, through the simultaneous analysis of lists of genes (gene panels) related to specific disease groups. Such gene panels can be either physical enrichment panels (for example using sequence capture), or be used as in silico lists for filtering applied to whole-exome or whole-genome sequencing data.

Unfortunately, NGS gene-panel-based strategies set up at the international level are currently extremely diverse, which complicates inter-laboratory exchange of data and expertise, and blurs the visibility of the diagnostic offers available for referring neurologists ("Where do I have to send my sample for each indication?"). At the European level, this problem has been addressed through recommendations underlining the importance of defining "core gene lists" [2] (genes for which disease-causing sequence variants are frequently implicated in the considered phenotype), and through international research projects [such as the FP7/2007-2013 European Community Seventh Framework Program projects NMD-chip (http://cordis.europa.eu/ result/rcn/56092_en.html) and NEUROMICS (https:// cordis.europa.eu/result/rcn/226821_en.html)], which illustrate that the inter-laboratory diversity of gene panels remains a reality in current medical practice in most countries of the European Union.

In France, an effort for the structuration of the genetic diagnosis for myopathies has been carried out since the creation of the French National Network for Rare Neuromuscular Diseases (Filière Nationale des Maladies Rares Neuromusculaires, FILNEMUS) in 2014, within the second National Plan for Rare Diseases.

In the field of rare diseases, among the 23 National Networks for Rare Disease recognized by the French Ministry of Health (http://solidarites-sante.gouv.fr/soinset-maladies/prises-en-charge-specialisees/maladies-rares/a rticle/l-offre-de-soins), FILNEMUS constitutes the second most important network in terms of active patient cohorts with more than 44,000 individuals followed in the nationwide 32 reference centers and 39 affiliated competence centers for neuromuscular disorders throughout France (the first being the National Network for Intellectual Deficiencies).

In 2016, a nationwide working group was initiated with the objective of homogenizing the genetic diagnostic strategies using NGS for myopathies in France, and taking into account the "Guidelines for diagnostic next-generation sequencing" published by the EJHG [2] to establish specific consensual "disease gene lists", allowing for "uniformity in testing between different laboratories and ensuring equity of testing for patients from different regions".

\section{Discussion}

A consultation of the nine French genetic diagnosis laboratories using NGS for myopathies has allowed to identify consensual key points as the foundation for the nationwide standardization: giving a central importance to the clinical entry-diagnosis; maintaining the existing nationwide distribution of expertise for specific groups of myopathies, while allowing for progressive extension of local expertise towards novel domains to favor local joint clinical-genetic board conclusions; taking into account the existing NGS platform capacities of the participating laboratories; and developing an efficient analysis turn-over time.

A consensual diagnostic strategy has been set up, based on the determination of 13 clinical and/or histological entrydiagnosis groups (Supplementary table 1)(Limb Girdle Muscular Dystrophies, Congenital Myopathies nemalinepositive, Congenital Myopathies nemaline-negative, Foetal and Neonatal Arthrogryposes, Congenital Muscular Dystrophies-except alpha-dystroglycanopathies, Alphadystroglycanopathies, Distal and Scapuloperoneal Myopathies, Retractile Myopathies, Myofibrillar Myopathies and Inclusion Body Myopathies, Congenital Myasthenias, Periodic Paralysis, Non-dystrophic Myotonia, and Metabolic Myopathies).

For each clinical and/or histological entry-diagnosis group, corresponding implicated genes were selected based on the following criteria: classification within the Gene Table of Neuromuscular Disorders [1]; routine diagnostic practice of the nine French genetic diagnosis laboratories using NGS for myopathies; and review of bibliographic evidence for the implication of the genes in the respective entry-diagnosis groups, categorized as "convincing" through at least two independent reports of causal mutations of the genes in the respective entry-diagnosis groups, associated to one report of functional evidence, or as "limited" in case of insufficient available bibliographic documentation. A total of 199 genes have been included within the different consensual gene lists.

For the different clinical and/or histological entrydiagnosis groups, the strategy consists either in a successive NGS analysis of a "core gene list" followed in case of a negative result by the analysis of an "exhaustive gene list" (successive analysis of firstly the known most prevalently implicated genes for the respective group of myopathies, followed by the analysis of all remaining known genes for the respective group); or in the NGS-analysis of a "unique exhaustive gene list" (all known genes for the respective 


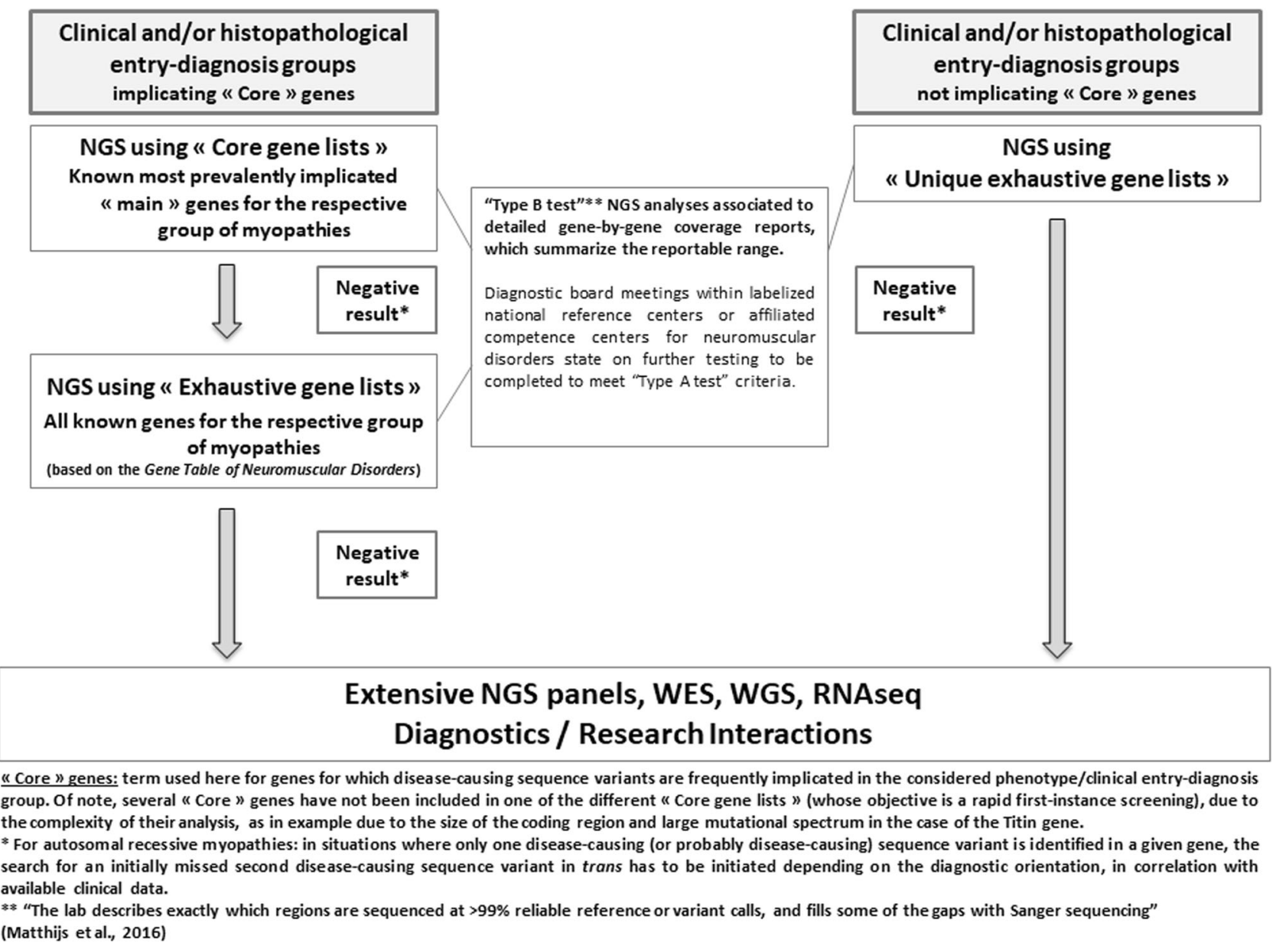

Fig. 1 National French consensus strategy for genetic diagnosis of myopathies using NGS

group of myopathies, in case no "main", particularly prevalent genes (i.e., "core" genes) are known to be implicated in the respective group) (Fig. 1). The accurate initial determination of the patient's entry-diagnosis group is a key point in this strategy, to optimize genetic screening of the most relevant genes. Noteworthy, in case the clinician is confronted with a situation where the entry-diagnosis group is difficult to define, warranting differential diagnosis, a simultaneous ordering and analysis of several of the consensual gene lists may be undertaken (for example, a difficulty in determining an entry-diagnosis group of "Congenital Myopathies nemaline-positive" or "Congenital Myopathies nemaline-negative" if insufficient histological data are available, would lead to the simultaneous analysis of both gene lists).

Each gene included in any of the lists has to be sequenced using NGS with specifications meeting the "Type B test" criteria [2], in conjunction with a detailed gene-by-gene coverage report, which summarizes the reportable range (i.e., the portion of the clinical target for which reliable calls have been generated) [2]. In case no genetic diagnosis is obtained following NGS-analysis, diagnostic board meetings among clinicians and geneticists, within the respective labelized national reference centers or affiliated competence centers for neuromuscular disorders, will state on a patient-by-patient basis, which additional Sanger testing has to be completed to meet "Type A test" criteria (if not already reached during the initial testing) for the analyzed sample, depending on available clinical data, knowledge on the genes' mutational spectra, and review of the literature. This strategy allows for immediate operationability, while taking into account the guidelines, and obviously keeping in mind the objective of meeting "Type A test" criteria for most if not all genes with the improvement of NGS technologies.

This consensual diagnostic strategy has been validated by an expert clinician group, and is currently being adopted by all participating laboratories throughout France, including the determination of expert laboratories for the different thematics. The role of expert laboratories includes the definition of the "exhaustive gene list" (based on their technical and theoretical expertise in the analysis of specific 
gene panels), and also, when required, their involvement in functional validation of problematic sequence variants. They may be consulted for data exchange and whenever prenatal genetic testing is required in their field of expertise.

Each laboratory declares its respective lists analyzed, on the FILNEMUS website (http://www.filnemus.fr/), therefore clarifying the nationwide diagnostic offer. Moreover, the standardization will allow for efficient data exchange between laboratories, including consensual variant classifications and inter-laboratory external quality assessments.

Through the nationwide FILNEMUS genetic diagnosis working-group, the consensual gene lists will be yearly updated, with the objective of progressively classifying all "gene-disease" associations according to the recently published ClinGen Clinical Validity framework [3]. These gene lists are being included in diagnostic flowcharts which are currently being developed within FILNEMUS.

Acknowledgements We sincerely thank Shahram Attarian, Christine Barnerias, Marc Bartoli, Anthony Behin, Claude Cances, Jean-Marie Cuisset, Andoni Echaniz-Laguna, Bruno Eymard, Svetlana Gorokhova-Devred, Pascal Laforet, Nicolas Levy, Armelle Magot, Philippe Petiot, Susana Quijano Roy, François Rivier, Sabrina Sacconi, Tanya Stojkovic, and Guilhem Sole for their contribution in reviewing the definite gene list documents. We sincerely thank Anne Barlier,
Gisèle Bonne, Claude Jardel, Delphine Lacourt, Philippe Latour, Vincent Procaccio, Pascale Richard, and Pascale Saugier-Veber for organizational discussions. This study was supported by the Filière Nationale des Maladies Rares Neuromusculaires FILNEMUS, AFMTELETHON, and by a Grant FP7/2007-2013 from the European Community Seventh Framework Program (Grant Agreement No. 2012-305121) "Integrated European omics research project for diagnosis and therapy in rare neuromuscular and neurodegenerative diseases" (NEUROMICS).

\section{Compliance with ethical standards}

Conflict of interest The authors declare that they have no conflict of interest.

\section{References}

1. Bonne G, Rivier F, Hamroun D. The 2018 version of the gene table of monogenic neuromuscular disorders (nuclear genome). Neuromuscul Disord. 2017;27:1152-83.

2. Matthijs G, Souche E, Alders M, Corveleyn A, Eck S, Feenstra I, et al. Guidelines for diagnostic next-generation sequencing. Eur J Hum Genet. 2016;24:2-5.

3. Strande NT, Riggs ER, Buchanan AH, Ceyhan-Birsoy O, DiStefano $\mathrm{M}$, Dwight SS, et al. Evaluating the clinical validity of gene-disease associations: an evidence-based framework developed by the clinical genome resource. Am J Hum Genet. 2017;100:895-906. 